\title{
Localizing predicate activity in resolution proofs
}

\author{
Marcello Sachs
}

September 1, 2019

\section{Introduction}

It is known that resolution-derivation of statements in conjunctive-normal-form is both sound and refutation-complete for Classical First Order Logic [1]. This provides a different angle for proving things about derivability in that language. This paper makes an approach along this line. A theorem is (constructively) proven regarding the existence of resolution-derivations satisfying certain structural properties, and this theorem is then used to offer a new constructive proof of an important result regarding Classical First Order Logic.

More specifically, a theorem is shown which implies that given any resolutionderivation $D_{1}$ and set of predicate-terms $S P_{1}$, a resolution-derivation $D_{2}$ can be constructed whose axiomatic basis is a subset of that of $D_{1}$, whose resultclause is the same as that of $D_{1}$, and whose every step involving an elimination of a literal with a term in $S P_{1}$ is such that every step leading to it also involves an elimination of a literal with a term in $S P_{1}$. This implication of the theorem is then used to provide a new constructive proof of the Craig Interpolation Theorem [2] for Classical First Order Logic.

I'll begin by laying out the relevant terminology, and then establishing a set of lemmas. Following this is the statement and proof of the central theorem of the paper, called a localization theorem because of the way in which it allows us to localize the activity of a given set of predicates. Finally, the new constructive proof of Craig's Interpolation Theorem is provided.

\section{Terminology}

\subsection{Concepts in the field}

Definition 1. resolution-proof: By this we will mean a conjunctive-normalform resolution proof in Classical First Order Logic. Such a proof involves a set of steps, where the input to each step is either a clausal representation of an axiom of some theory, or the output of some other step. If we say that a step involves the elimination of a term $t$, we mean by this that a literal eliminated at that step includes $t$. 
Definition 2. variable-substitution: In resolution-proofs, it is valid under certain circumstances to, for a certain step, transform the literals from an input so that certain variables are replaced with certain other terms (these other terms being variables, constants, or skolem-functions). We will refer to the function specifying such replacements for a given step as a variable-substitution.

Definition 3. valid-resolution-step: A step in a resolution-proof involves the elimination of a set of literals. Let us define here the conditions under which that elimination is valid, so we can reference them later in proofs. It will suffice for our purposes to only define validity for the case that a step involves no variable-substitutions. Let $O_{1}$ be the output clause for a step $S_{1}$ that involves no variable-substitutions, and $I_{1}, I_{2}$ its two input clauses. Then $S_{1}$ is valid iff there exists a set $E$ such that :

(i) $E$ is a subset of $I_{1} \cup I_{2}$.

(ii) $E$ includes at most one element from each of $I_{1}, I_{2}$

(iii) If $E$ includes two elements, they are complements

(iv) $O_{1}=\left\{I_{1} \cup I_{2}\right\}-E$

Definition 4. tree: This refers to the usual graph-theoretic concept - a Directed Acyclic Graph where every node is pointed to by at most one other. If a node $n_{1}$ points to $n_{2}$, we say that $n_{1}$ is $n_{2}$ 's parent, and $n_{2}$ is $n_{1}$ 's child. A node's descendants are all those nodes reachable by paths pointing away from it, and a node's ascendants are all those nodes for which it is an descendant. The inclusive-descendants of a node is the set including that node and its descendants, and inclusive-ascendants is defined analogously. A root is a node with no parents, and a leaf is a node with no children. If, in the context of a tree $t_{1}$, we speak of that sub-tree $t_{2}$ of it which is rooted by a node $n_{1}$, we mean that sub-tree of $t_{1}$ consisting of $n_{1}$ 's inclusive-descendants, and all of the edges which point among them.

Definition 5. resolution-tree: There is a natural way to determine a tree based on a resolution-proof, and we will call such a tree a resolution-tree. First, create a single node, and associate it to the final-clause of the proof. If the final step of the proof had two (as opposed to zero) inputs, then add two nodes to the tree, associate each node to each input, respectively, and establish edges making the original node their parent. Continue doing this iteratively until the only leaf-nodes in the tree are those associated to axiomatic inputs (inputs that are not the output of any step in the proof). Note that a given clause may come to be associated to more than one node. Also note that the tree will have a single root.

\subsection{Concepts specific to this paper}

Definition 6. abstractly-equivalent: Two resolution-trees $t_{1}$ and $t_{2}$ are abstractly-equivalent if a graph-isomorphism $f_{1}$ exists from $t_{1}$ to $t_{2}$ such 
that, for any node $n_{1}$ in $t_{1}$, the clause $c_{1}$ associated to $n_{1}$ and the clause $c_{2}$ associated to $f_{1}\left(n_{1}\right)$ are such that they differ from each other only in regards their variables and constants.

Definition 7. complete-concretization: The complete-concretization $t_{2}$ of a resolution-tree $t_{1}$ is the tree produced by altering the clause $c_{1}$ associated to each node $n_{1}$ in the following way : send $c_{1}$ through the composition of variable-substitutions which exist between $n_{1}$ and $t_{1}$ 's root. It can be seen that $t_{2}$ will be a valid resolution-tree that involves no variable-substitutions, and which has the same result-clause as $t_{1}$.

Definition 8. abstract-rebasis: Given a set of clauses $X_{1}$, and a tree $t_{1}$ such that every clause $c_{1}$ associated to a leaf-node $n_{1}$ in $t_{1}$ results from a (valid) variable-substitution operation on some clause $c_{2}$ in $X_{1}$, let the abstract-rebasis of $t_{1}$ be the tree resulting from taking $t_{1}$, and changing each leaf node so that instead of being associated to its original clause, it is now associated to the relevant matching clause in $X_{1}$. Clearly, the resulting tree has the same resultclause as the original, is a valid resolution-tree, and is abstractly-equivalent to the original.

Definition 9. organizing-condition: A condition $C_{\text {org }}$ on nodes of a resolutiontree such that :

(i) $C_{\text {org }}$ is defined relative to a given set $S P_{1}$ of predicate terms

(ii) $C_{\text {org }}$ is satisfied by every leaf-node whose clause includes a term in $S P_{1}$

(iii) For any three nodes $n_{1}, n_{2}, n_{3}$, where $n_{3}$ is parent to $n_{1}$ and $n_{2}$ , and $n_{3}$ involves an elimination in $S P_{1}: n_{1}$ and $n_{2}$ satisfy $C_{\text {org }}$ iff $n_{3}$ does.

(iv) If a node does not satisfy $C_{\text {org }}$, then neither does its parent

(v) For any two nodes $n_{1}$ and $n_{2}$, if the sub-trees rooted by $n_{1}$ and $n_{2}$, respectively, are abstractly-equivalent, then $n_{1}$ satisfies $C_{l o c}$ iff $n_{2}$ does

Two examples of this kind of condition are :

- For a given set of predicate-terms $S P_{1}$, the condition that every leaf-node in one's inclusive-descendants includes a term in $S P_{1}$.

- For a given set of predicate-terms $S P_{1}$, the condition that every leafnode in one's inclusive-descendants includes a term in $S P_{1}$, and every non-leaf-node in one's inclusive-descendants involves the elimination of a literal that includes a term in $S P_{1}$.

Definition 10. localizing-condition: A condition $C_{l o c}$ on nodes of a resolutiontree such that :

(i) $C_{l o c}$ is defined relative to a given organizing-condition $C_{o r g}$ 
(ii) if $S P_{1}$ is the set of predicate-terms that $C_{\text {org }}$ is defined in relation to, then a node satisfies $C_{l o c}$ iff it (one or both of the following) satisfies $C_{\text {org }}$ , or is such that its elimination involves no term in $S P_{1}$.

Definition 11. localized-form: For a given localizing-condition $C_{l o c}$, a localizedform of a resolution-tree $t_{x}$ is any resolution-tree whose nodes all satisfy $C_{l o c}$, whose root node is associated to the same clause as that of $t_{x}$, and whose leaf nodes are associated to a set of clauses which is a subset of the set of clauses associated to the leaf nodes of $t_{x}$.

\subsection{Algorithms specific to this paper}

Note : the algorithms below produce trees whose nodes are associated to clauses. They may not always produce trees that represent valid resolution-proofs. However, our later work will rely only on the fact that they produce a tree representing a valid resolution-proof when called on particular inputs. What these inputs are will be specified later, and the fact of the output being valid in that case will be proven later. For now we will still refer to their outputs as resolution-trees, keeping in mind that the validity of these trees is to be shown later for particular cases.

Definition 12. tree_producer_1 $\left(t_{x}, t_{y}, n_{x}, n_{y}, L S_{x}\right)$ : For $n_{x}$, $n_{y}$ nodes in resolution-trees $t_{x}, t_{y}$, and $L S_{x}$ a set of literals, return a new resolution-tree constructed by :

1. creating a node and assigning it the clause consisting in the union of the literals in $n_{x}$ and $n_{y}$ 's clauses, minus the literals in $L S_{x}$

2. taking the sub-trees rooted by $n_{x}$ and $n_{y}$ in $t_{x}$ and $t_{y}$, respectively, and adding them to this new tree, and making the roots of these sub-trees children to the node created in part 1.

Definition 13. tree_producer_2( $\left.t_{x}, t_{y}, n_{x}, N_{y}, L S_{x}\right)$ : For $n_{x}$ a node in a resolution-tree $t_{x}, N_{y}$ a set of nodes in resolution-tree $t_{y}$, none of which are ascendant of any other, and $L S_{x}$ a set of literals : return the tree which results from taking $t_{y}$, replacing every sub-tree rooted by a node $n_{y}$ in $N_{y}$ with tree_producer_1( $\left.t_{x}, t_{y}, n_{x}, n_{y}, L S_{x}\right)$, and altering the clause-assignment for each ascendant $n_{a}$ of such a replacement so that, if it was $c_{1}$ beforehand, it is now the union of the literals in $c_{1}$ and in the clause assigned to $n_{x}$, minus $L S_{x}$.

Definition 14. tree_producer_3( $\left.t_{x}, t_{x}, N_{x}, N_{y}, L S_{x}\right)$ : For sets $N_{x}, N_{y}$ of nodes from resolution-trees $t_{x}, t_{y}$, respectively, where no node in the set $\left\{N_{x} \cup N_{y}\right\}$ is ascendant of another, and a set of literals $L S_{x}$ , return the tree constructed in the following way : begin with $t_{x}$, and then replace each sub-tree rooted by a node $n_{x}$ in $N_{x}$ with tree_producer_2( $\left.n_{x}, N_{y}, L S_{x}\right)$. Then, for each ascendant $n_{a}$ of a replacement, alter its clause assignment so that if it was $c_{1}$ beforehand, it is now the union of the literals in $c_{1}$ and in the clause assigned to $t_{y}$ 's root, minus $L S_{x}$. 


\section{On localizing-conditions}

Lemma 3.1. If resolution-trees $t_{1}$ and $t_{2}$ are abstractly-equivalent, then $t_{1}$ 's root satisfies a localizing-condition $C_{l o c}$ iff $t_{2}$ 's root does.

Proof. Let $C_{\text {org }}$ be the organizing-condition in relation to which $C_{l o c}$ is defined, and $S P_{1}$ the set of predicate-terms in relation to which $C_{o r g}$ is defined. Suppose $t_{1}$ 's root satisfies $C_{l o c}$. Then either (one or both of the following) the elimination at $t_{1}$ 's root does not involve a term in $S P_{1}$, or $t_{1}$ 's root satisfies $C_{\text {org }}$. In the former case, given the nature of abstract-equivalence, we know that the elimination at $t_{2}$ 's root will likewise not involve a term in $S P_{1}$. In the latter case, given part (v) of Definition 9 of organizing-condition, we know that $t_{2}$ 's root will also satisfy $C_{\text {org }}$. The same reasoning works for showing that if $t_{2}$ 's root satisfies $C_{l o c}$, then so will $t_{1}$ 's root.

Lemma 3.2. For any localizing-condition $C_{l o c}$ and resolution-tree $t_{1}$, if a sub-tree st $t_{1}$ of $t_{1}$ whose nodes do not all satisfy $C_{l o c}$ is replaced by a sub-tree whose root is associated to the same clause as $s_{1}$ 's root, then any non-replaced node in $t_{1}$ that satisfied $C_{l o c}$ beforehand will do so afterwards as well.

Proof. Let $C_{\text {org }}$ be the organizing-condition that $C_{l o c}$ was defined in relation to, and $S P_{1}$ the set of predicate-terms that $C_{\text {org }}$ was defined in relation to. Given Definition 10 of localizing-condition, the fact that some nodes in the replaced sub-tree did not satisfy $C_{l o c}$ implies that some nodes in the replaced sub-tree did not satisfy $C_{\text {org }}$.

Given that, by part (iv) of Definition 9 of organizing-condition, a parent of a node that doesn't satisfy such a condition cannot itself satisfy such a condition, we know that all the ascendants of the replaced sub-tree could not have satisfied $C_{\text {org }}$ beforehand.

Thus, any ascendant of the replaced sub-tree which satisfied $C_{l o c}$ beforehand must have been such that the step it represents does not involve the elimination of a literal with a term in $S P_{1}$. The replacement does not effect which steps these nodes represent, so any of these nodes which satisfied $C_{l o c}$ beforehand will continue to do so afterwards.

As far as the nodes which were neither replaced nor ascendant to a replacement - that $C_{l o c}$ satisfaction is preserved among such nodes follows from Lemma 3.1 , and the fact that each is the root of a sub-tree which is abstractly-equivalent to itself.

Lemma 3.3. For every localizing-condition $C_{l o c}$, defined in relation to an organizing-condition $C_{\text {org }}$ and set of predicate-terms $S P_{1}$, and every pair of nodes $n_{1}, n_{2}$ in a resolution-tree that involves no variable-substitutions:

If

- $n_{2}$ is an ascendant of $n_{1}$

- $n_{2}$ 's child that is not an ascendant of $n_{1}$ has a clause that includes a literal lit $_{1}$ with a term in $S P_{1}$ 
- $n_{2}$ 's clause does not include lit 1

- For $N_{x}$ the set of nodes between (non-inclusive) $n_{1}$ and $n_{2}$, none of the nodes in $N_{x}$ satisfy $C_{\text {org }}$, but they all satisfy $C_{l o c}$

Then $n_{1}$ 's clause does not include lit 1 .

Proof. Since the nodes in $N_{x}$ satisfy $C_{l o c}$ but not $C_{\text {org }}$, it must be that none of them involve an elimination with a term in $S P_{1}$. This entails that $l i t_{1}$ cannot be eliminated in one of those nodes. Suppose that $n_{1}$ includes $l_{i t}$. This would then imply that both of $n_{2}$ 's children include $l i t_{1}$, while $n_{2}$ does not. This would render the resolution-step that $n_{2}$ represents invalid, contradicting the assumption that it comes from a resolution-tree.

Lemma 3.4. For every localizing-condition $C_{l o c}$, defined in relation to an organizing-condition $C_{\text {org }}$ and set of predicate-terms $S P_{1}$, and every node $n_{1}$ in a resolution-tree that involves no variable-substitutions, and every literal lit ${ }_{1}$ which includes a term in $S P_{1}$ :

If

- $n_{1}$ 's parent does not satisfy $C_{\text {org }}$

- no member $n_{d}$ of $n_{1}$ 's inclusive-descendants is such that all the following are true of it: $n_{d}$ satisfies $C_{\text {org }}$, it's parent does not, and $n_{d}$ 's clause includes lit 1

Then $n_{1}$ 's clause does not include lit 1 .

Proof. Suppose lit $_{1}$ is in $n_{1}$ 's clause. Then there must be some leaf node $n_{2}$ which is in the inclusive-descendants of $n_{1}$, whose clause includes lit $t_{1}$, and where $l i t_{1}$ is not eliminated between $n_{2}$ and $n_{1}$. Given part (ii) of Definition 9 of organizing-condition, we know that $n_{2}$ satisfies $C_{\text {org }}$. And so there will be some node $n_{x}$, which is either $n_{2}, n_{1}$, or some node in between, which includes $l i t_{1}$, satisfies $C_{\text {org }}$, and is such that its parent does not satisfy $C_{\text {org }}$. Since $n_{x}$ is in $n_{1}$ 's inclusive-descendants, this contradicts the second antecedent.

\section{On localized-forms}

Lemma 4.1. For any resolution-tree $t_{1}$ and localizing-condition $C_{l o c}$ : if a sub-tree st $t_{1}$ of $t_{1}$ whose only $C_{l o c}$-violating node is its root is replaced by a localized-form of st $_{1}$, this produces a tree $t_{2}$ where:

- $t_{2}$ 's root node is associated to the same clause as that of $t_{1}$

- the set of clauses associated to $t_{2}$ 's leaf-nodes is a subset of the set of clauses associated to $t_{1}$ 's leaf-nodes

- $t_{2}$ has strictly fewer nodes than $t_{1}$ that do not satisfy $C_{l o c}$ 
Proof. The first two points should be clear simply from the nature of trees and Definition 11 of localized-form. The last point follows from Lemma 3.1 and 3.2.

Lemma 4.2. For every localizing-condition $C_{l o c}$, and every resolution-tree $t_{1}$ whose every node satisfies $C_{l o c}$ except for its root, if $t_{1}$ involves no variablesubstitutions, then we can construct a localized-form $t_{2}$ of $t_{1}$ which also involves no variable-substitutions.

Proof. Let $C_{\text {org }}$ be the organizing-condition that $C_{l o c}$ is defined in relation to, and $S P_{1}$ the set of predicate terms that $C_{\text {org }}$ is defined in relation to. Since $t_{1}$ 's root does not satisfy $C_{l o c}$, it must involve the elimination of a literal $l_{i t}$ whose predicate term is in $S P_{1}$. Let $n_{\text {main }}$ be the child of $t_{1}$ whose clause includes $l i t_{1}$, and $n_{\text {other }}$ its other child. Let $L S_{1}$ be the set of literals eliminated at $t_{1}$ 's root (must consist of $l_{i} t_{1}$ alone or $l i t_{1}$ and its complement, lit ${ }_{1}^{c}$ ). Let $N_{1}$ be the subset of $n_{\text {main }}$ 's inclusive-descendants such that, for each $n_{1}$ in $N_{1}$ :

- $n_{1}$ 's clause includes $l i t_{1}$

- lit $_{1}$ does not get eliminated between $n_{1}$ and $n_{\text {main }}$

- $n_{1}$ satisfies $C_{\text {org }}$

- $n_{1}$ 's parent does not satisfy $C_{o r g}$

Define $N_{2}$ analogously for $n_{\text {other }}$ and $l i t_{1}^{c}$. Let $s t_{\text {main }}$ and $s t_{\text {other }}$ be the sub-trees rooted by $n_{\text {main }}$ and $n_{\text {other }}$, respectively. Let $t_{3}=$ tree_producer_3( $\left.s t_{\text {main }}, s t_{\text {other }}, N_{1}, N_{2}, L S_{1}\right)$. Note that the inputs to this call to the function satisfy the function's input-requirements (for example, that $\left\{N_{1} \cup N_{2}\right\}$ should include no member which is ascendant of another.) The rest of our proof will involve showing that $t_{3}$ is the $t_{2}$ whose existence is claimed by the lemma.

Basis Preservation That the set of clauses associated to $t_{3}$ 's leaf nodes is a subset of that for $t_{1}$ follows from the fact that the leaf nodes produced in the output of the function are all copied from its input trees, which are both sub-trees of $t_{1}$.

Result Preservation By definition, the clause associated to $t_{1}$ 's root is the union of the literals in $n_{\text {main }}$ 's clause and the literals in $n_{\text {other }}$ 's clause, minus $L S_{1}$. So let us show this will also be true for $t_{3}$ 's root-node. Consider the following two cases:

1. $N_{1}$ includes $n_{\text {main }}$ Given how $N_{1}$ is defined, this implies that $N_{1}$ is simply $\left\{n_{\text {main }}\right\}$. This then implies that $N_{2}$ cannot include $n_{\text {other }}$. For if it did, then both children of $t_{1}$ 's root would satisfy $C_{\text {org }}$, making it so that $t_{1}$ 's root, which involves an elimination in $S P_{1}$, would satisfy $C_{\text {org }}$ (given part 
(iii) of Definition 9 of organizing-condition) and thus $C_{l o c}$. This contradicts the premise that $t_{1}$ 's root does not satisfy $C_{l o c}$. It can be seen that since $N_{2}$ does not include $n_{\text {other }}$, it must include at least one of its descendants.

Considering the definition of tree_producer_3, $t_{3}$ will be the output of the call tree_producer_2( $\left.s t_{\text {main }}, s_{\text {other }}, n_{\text {main }}, N_{2}, L S_{1}\right)$. By definition of tree_producer_2, the root node of the output to this call will be $n_{\text {other }}$, except with its clause assignment altered. The new clause to which it will be assigned can be seen from the definition of the algorithm to be precisely the union of the literals in its former clause and the literals in $n_{\text {main }}$ 's clause, minus $L S_{1}$.

2. $N_{1}$ does not include $n_{\text {main }}$ Given how $N_{1}$ is defined, this implies $N_{1}$ will include some node $n_{1}$ which is a descendant of $n_{\text {main }}$. This means the root node of $t_{3}$ will be $n_{\text {main }}$, except with its clause-assignment altered. The alteration can be seen, from the definition of the algorithm to produce precisely the clause needed.

Validity It is clear that the functions we defined produced trees where every node is assigned to a clause, but it may not be clear that these trees are resolution-trees - as in, that they represent valid resolution-proofs. We will now show that, when one provides the kind of inputs that we provide to the algorithms in our construction of $t_{3}$, they produce resolution-trees that represent valid resolution-proofs.

The call we make to tree_producer_3 in turn involves certain calls to tree_producer_2. In particular, these are calls of the kind tree_producer_2( $\left.s t_{\text {main }}, s t_{\text {other }}, n_{1}, N_{2}, L S_{1}\right)$, where $n_{1}$ is some node in $N_{1}$. A call of this kind in turn involves calls to tree_producer_1, of the kind tree_producer_1( $\left.s t_{\text {main }}, \quad s t_{\text {other }}, n_{1}, n_{2}\right)$, where $n_{1}$ and $n_{2}$ belong to $N_{1}, N_{2}$, respectively. So we begin by showing the validity of these 'sub-calls'. Nodes in sub-trees that are copied unaltered from $t_{1}$ will be valid given the assumed validity of $t_{1}$, so in the foregoing we will only concern ourselves with showing the validity of nodes that are not of that kind.

Validity of output of call to tree_producer_1 : There is only one node in this output which is not copied unaltered from $t_{1}$ - the root node $n_{r}$. Its two children, $n_{1}$ and $n_{2}$, which come from $N_{1}$ and $N_{2}$ respectively, have been copied unaltered (with the same clause assignments) from $t_{1} \cdot n_{r}$ 's clause is assigned to be the union of the literals in $n_{1}$ and $n_{2}$, minus $L S_{1}$. Recall that $L S_{1}$ consists of either $l i t_{1}$ or $l i t_{1}$ and its complement $l i t_{1}^{c}$. Given how $N_{1}$ and $N_{2}$ were defined, $n_{1}$ 's clause contains $l i t_{1}$, and $n_{2}$ 's clause contains $l i t_{1}^{c}$. What remains to be shown are two things. First, that $n_{2}$ 's clause does not contain $l i t_{1}$. This can be seen to follow from Lemma 3.3. Second, that if $L S_{1}$ includes $l i t_{1}^{c}$, then $n_{1}$ 's clause does not include $l i t_{1}^{c}$. This can also be seen to follow from Lemma 3.3.

Validity of output of call to tree_producer_2 : The only nodes in this output which are not copied unaltered from $t_{1}$ are those produced by calls to 
tree_producer_1 of the kind whose validity we just showed, and those copied from $t_{1}$ which are ascendant to nodes replaced by such calls, and have had their clauses altered. Let us consider an arbitrary node $n_{3}$ of the latter kind.

At least one of its children, $n_{3} c_{1}$, will either be the root of the output to the relevant kind of call to tree_producer_1, or it will be ascendant to such a node. In the former case, let $o n_{3} c_{1}$ be $n_{3}$ 's child in $t_{1}$ that was replaced by the call to tree_producer_1. In the latter case, let ${ }_{0} n_{3} c_{1}$ simply be $n_{3} c_{1}$ (except with its original clause assignment).

$n_{3}$ 's other child, $n_{3} c_{2}$, will either be of one of the two kinds that we mentioned $n_{3} c_{1}$ may be, or the root of a sub-tree copied unaltered from $t_{1}$. In the former case, define $o n_{3} c_{2}$ similarly to how we did $o n_{3} c_{1}$. In the latter case, let $o n_{3} c_{2}$ simply be $n_{3} c_{2}$. Note that we have designed the criteria for $o n_{3} c_{1}$ and on $_{3} c_{2}$ so that, in any case, both were children of $n_{3}$ in $t_{1}$.

Let's introduce some more terminology to simplify our reasoning. We will write $f_{c}\left(n_{x}\right)$ for a given node $n_{x}$ to represent the clause assigned to it. In the case that the clause assigned to a node is different in $t_{1}$ versus the output tree of the call to tree_producer_2, we will write $f_{c}\left(n_{x}\right.$, before $)$ vs. $f_{c}\left(n_{x}\right.$, after $)$ to distinguish.

Given our assumption that $t_{1}$ is valid and involves no variable-substitutions, we know that :

$$
f_{c}\left(n_{3}, \text { before }\right)=\left\{f_{c}\left(\text { on }_{3} c_{1}\right) \cup f_{c}\left(\text { on }_{3} c_{2}\right)\right\}-E_{1}
$$

where $E_{1}$ is some set of literals satisfying the conditions (i)-(iv) mentioned in Definition 3 of valid-resolution-step.

Given the definition of tree_producer_2 :

$$
f_{c}\left(n_{3}, \text { after }\right)=\left\{f_{c}\left(n_{3}, \text { before }\right) \cup f_{c}\left(n_{1}\right)\right\}-L S_{1}
$$

Recall that $n_{1}$ is one of the inputs to the kind of call to tree_producer_2 with which we are presently concerned, and that $n_{1}$ comes from $N_{1}$. If we combine the information from equation 1 and 2 :

$$
f_{c}\left(n_{3}, \text { after }\right)=\left\{\left\{\left\{f_{c}\left(\text { on }_{3} c_{1}\right) \cup f_{c}\left(\text { on }_{3} c_{2}\right)\right\}-E_{1}\right\} \cup f_{c}\left(n_{1}\right)\right\}-L S_{1}
$$

Given some simple reasoning regarding the nature of sets, this implies :

$$
\begin{aligned}
f_{c}\left(n_{3}, \text { after }\right)=\{ & \left.\left\{f_{c}\left(\text { on }_{3} c_{1}\right) \cup f_{c}\left(\text { on }_{3} c_{2}\right) \cup f_{c}\left(n_{1}\right)\right\}-L S_{1}\right\} \\
& -\left\{E_{1}-f_{c}\left(n_{1}\right)\right\}
\end{aligned}
$$

From the definition of tree_producer_2 and tree_producer_1:

$$
f_{c}\left(n_{3} c_{1}\right)=\left\{f_{c}\left(o_{3} c_{1}\right) \cup f_{c}\left(n_{1}\right)\right\}-L S_{1}
$$


When $n_{3} c_{2}$ is copied unaltered from $t_{1}$, clearly:

$$
f_{c}\left(n_{3} c_{2}\right)=f_{c}\left(o n_{3} c_{2}\right)
$$

and when this is not the case, given the definition of tree_producer_2 and tree_producer_1:

$$
f_{c}\left(n_{3} c_{2}\right)=\left\{f_{c}\left(\text { on }_{3} c_{2}\right) \cup f_{c}\left(n_{1}\right)\right\}-L S_{1}
$$

What we will try to show now (for reasons that will be clear later) is that none of the literals in $L S_{1}$ will be in $f_{c}\left(n_{3} c_{2}\right)$. Given equations 6 and 7 , this amounts to showing that, in the case that $n_{3} c_{2}$ is copied unaltered from $t_{1}$, none of the literals in $L S_{1}$ are in $f_{c}\left(o_{3} c_{2}\right)$. Recall that $L S_{1}$ consists either only of $l i t_{1}$ or of $l i t_{1}$ and $l i t_{1}^{c}$

Since $n_{3}$ is ascendant of a node which gets replaced by a call to tree_producer_1, we know (given the definition of tree_producer_2) that $n_{3}$ was ascendant to a member of $N_{2}$ in the original tree $t_{1}$. Given the definition of $N_{2}$, and part (iv) of Definition 9 of organizing-condition, this implies that $n_{3}$ does not satisfy $C_{o r g}$. If $n_{3} c_{2}$ is copied unaltered from $t_{1}$, we know from this that (given the definition of tree_producer_2) no member of $N_{2}$ is in the inclusive-descendants of $\mathrm{on}_{3} \mathrm{c}_{2}$. It can be seen that these two facts allow us to apply Lemma 3.4 to show that, in this case, the potential member $l i t_{1}^{c}$ of $L S_{1}$ will not be in $f_{c}\left(o_{3} c_{2}\right)$.

To show that the member $l i t_{1}$ of $L S_{1}$ will not be in $f_{c}\left(o n_{3} c_{2}\right)$ (in the case that $n_{3} c_{2}$ is copied unaltered from $\left.t_{1}\right)$, consider the following:

- Given that $n_{3}$ was an ascendant of a node in $N_{2}$ in $t_{1}$, and $n_{3}$ was ${ }_{o n} c_{2}$ 's parent, we know that none of the nodes between ${ }^{o n} n_{3} c_{2}$ and $n_{r}$ in $t_{1}$ satisfied $C_{\text {org }}$.

- Given that $n_{3}$ was, in $t_{1}$, within a sub-tree rooted by a child of $t_{1}$ 's root $n_{r}$, and that $t_{1}$ 's only $C_{l o c}$ violating node was $n_{r}$, we know that all the nodes between $o n_{3} c_{2}$ and $n_{r}$ in $t_{1}$ satisfied $C_{l o c}$.

- $n_{r}$, an ascendant of $o n_{3} c_{2}$, does not include $l_{i t_{1}}$ ( $l i t_{1}$ was originally defined as a literal which is eliminated at $n_{r}$ ).

- $n_{r}$ 's child that is not an ascendant of $o n_{3} c_{2}$ is $n_{\text {main }}$, whose clause includes $l_{i t}$ (this is precisely how $n_{\text {main }}$ was defined).

- The above points allow us to apply Lemma 3.3 to show that ${ }^{\circ} n_{3} c_{2}$ 's clause will not include $l_{i t_{1}}$

Now that we have shown that none of the literals in $L S_{1}$ will be in $f_{c}\left(n_{3} c_{2}\right)$, we can combine equations 5, 6 and 7 in the following way:

$$
f_{c}\left(n_{3} c_{1}\right) \cup f_{c}\left(n_{3} c_{2}\right)=\left\{f_{c}\left(\text { on }_{3} c_{1}\right) \cup f_{c}\left(\text { on }_{3} c_{2}\right) \cup f_{c}\left(n_{1}\right)\right\}-L S_{1}
$$


And now we can combine 4 and 8 to yield :

$$
f_{c}\left(n_{3}, \text { after }\right)=\left\{f_{c}\left(n_{3} c_{1}\right) \cup f_{c}\left(n_{3} c_{2}\right)\right\}-\left\{E_{1}-f_{c}\left(n_{1}\right)\right\}
$$

Let $E_{2}$ stand for $\left.\left\{E_{1}-f_{c}\left(n_{1}\right)\right\}\right)$. What remains to be shown is that $E_{2}$ satisfies the conditions (i)-(iv) mentioned in Definition 3 of valid-resolution-step. We prove each of these in turn below. Before we begin, note the following:

Note 1. $E_{1}$ does not intersect $L S_{1}$. To see this, consider the following. Given the definition of tree_producer_2, we know that $n_{3}$ was ascendant, in $t_{1}$, to some node in $N_{2}$. Given the definition of $N_{2}$, this implies that $n_{3}$ did not satisfy $C_{\text {org }}$ in $t_{1}$. Given that $t_{1}$ 's only $C_{l o c}$ violating node is its root, and that $n_{3}$ comes from a sub-tree rooted by a child of $t_{1}$ 's root, we know that $n_{3}$ must have satisfied $C_{l o c}$ in $t_{1}$. Since $n_{3}$ satisfied $C_{l o c}$ but not $C_{\text {org }}$, it cannot have involved an elimination of a term in $S P_{1}$, and thus cannot have involved the elimination of any literal in $L S_{1}$.

And now for demonstrating the satisfaction of each part of the definition of valid-resolution-step (each demonstration is labelled by the index of the part of the definition it relates to) :

(i) By definition, $E_{1}$ is a subset of $\left\{f_{c}\left(o n_{3} c_{1}\right) \cup f_{c}\left(o n_{3} c_{2}\right)\right\}$. From equation 8 and Note 1 , we then know that $E_{1}$ is a subset of $\left\{f_{c}\left(n_{3} c_{1}\right) \cup f_{c}\left(n_{3} c_{2}\right)\right\}$. Since $E_{2}$ defined as a subset of $E_{1}$, we are done.

(ii) Suppose $l_{i t}$ is an element in $E_{2}$. It is then in $E_{1}$, and since $t_{1}$ is valid, this means it is in one of $f_{c}\left(o n_{3} c_{1}\right)$ or $f_{c}\left(o n_{3} c_{2}\right)$, and not the other. Suppose it is in the former (and thus not in the latter). Given equation 5 and note 1 , this means it is also in $f_{c}\left(n_{3} c_{1}\right)$. Given equation 6 and 7 , it is not in $f_{c}\left(n_{3} c_{2}\right)$ as long as it is not in $f_{c}\left(n_{1}\right)$. That it is not in $f_{c}\left(n_{1}\right)$ follows simply from the fact that the formula we used to define $E_{2}$ to begin with was $\left\{E_{1}-f_{c}\left(n_{1}\right)\right\}$. Similar reasoning works for the case where lit $t_{x}$ is in $f_{c}\left(\mathrm{on}_{3} c_{2}\right)$.

(iii) Follows from fact that $E_{2}$ is a subset of $E_{1}$, and $t_{1}$ is valid.

(iv) This is precisely the content of equation 9

Validity of output of call to tree_producer_3 : The only nodes in the output of this call that aren't copied unaltered from $t_{1}$ are outputs from calls to tree_producer_2 of the kind whose validity we just showed, and nodes which are ascendant of nodes that have been thus replaced - these nodes being copied over from $t_{1}$ but having their clause assignment altered.

Showing the validity of these latter kinds of nodes can be done in a way very similar to the way we showed validity for the analogous nodes (nodes ascendant of replacements) in the output of the relevant kind of call to tree_producer_2. 
For each such node $n_{4}$, its two children $n_{4} c_{1}$ and $n_{4} c_{2}$ in the output will stand in an analogous relation towards two nodes on $c_{1}$ and on $c_{2}$ as $n_{3} c_{1}$ and $n_{3} c_{2}$ did towards the nodes $o_{3} c_{1}$ and $o_{3} c_{2}$ we defined in the earlier proof.

The proof then runs analogously to the way it did for the tree_producer_2 case, with every string " $n_{3}$ " replaced with " $n_{4}$ ", and " $n_{1}$ " with " $n_{\text {other }}$ ".

Satisfaction of $C_{l o c}$ We can divide the nodes of $t_{3}$ into three kinds of nodes - those which are the roots of sub-trees simply copied from $t_{1}$, those which are the roots of trees produced by the relevant call to tree_producer_1, and those which are ascendant to nodes of the second kind and have had their clause-assignments changed.

We know all the nodes of the first kind will satisfy $C_{l o c}$ because they were descendants of $t_{1}$-root, and since $t_{1}$ 's only $C_{l o c}$ violating node is its root, this means they must have satisfied $C_{l o c}$ beforehand. From Lemma 3.1, they will continue to do so in $t_{3}$.

That a node $n_{x}$ of the second kind will satisfy $C_{l o c}$ follows from the following considerations. Its children come (unaltered) from $\left\{N_{1} \cup N_{2}\right\}$, which all satisfied $C_{\text {org }}$ in $t_{1}$. Given part (v) of Definition 9 of organizing-condition, those children will continue to satisfy $C_{\text {org }}$ in $t_{3}$. Finally, $n_{x}$ by definition involves the elimination of literals in the non-empty set $L S_{1}$, which include a predicate-term from $S P_{1}$. Given part (iii) of the definition of organizingcondition, these facts together imply that $n_{x}$ will satisfy $C_{o r g}$, and thus $C_{l o c}$.

That a node of the third kind will satisfy $C_{l o c}$ follows from the following considerations. In the work showing the validity of $t_{3}$, it became apparent that the elimination $E_{2}$ which occurs at every such node in $t_{3}$ is a subset of the elimination $E_{1}$ that used to occur in it in $t_{1}$ (this was shown directly for nodes within the output to the relevant kind of calls to tree_producer_2, and would be seen to be true for all other nodes ascendant of replacements in the proof for the validity of the output of the relevant call to tree_producer_3 which we described would run in a manner quite analogous to that for the call to tree_producer_2). This means that if $E_{2}$ includes a term in $S P_{1}$, then so would $E_{1}$ have. We also know that since such a node used to be ascendant of a node either in $N_{1}$ or $N_{2}$, it must have not originally satisfied $C_{\text {org }}$ in $t_{1}$ - Thus if $E_{2}$ includes a term in SP1, the node would not have satisfied $C_{l o c}$ in $t_{1}$. However, it can be seen that the node must have satisfied $C_{l o c}$ in $t_{1}$. For consider that none of these nodes were the root of $t_{1}$, and that $t_{1}$ 's only $C_{l o c}$ -violating node is its root. Since the supposition of $E_{2}$ including a term in $S P_{1}$ leads to a contradiction, we know that it can't have included such a term. From Definition 10 of localizing-condition, this implies that this node satisfies $C_{l o c}$ in $t_{3}$.

No Variable-Substitutions That $t_{3}$ involves no variable-substitutions is demonstrated by the fact that in proving the validity of nodes of $t_{3}$ which were not copied unaltered from $t_{1}$, we relied only upon a definition of validresolution-step (Definition 3) pertinent to resolution-proofs that involve no variable- 
substitutions. As for the nodes which were copied unaltered from $t_{1}$, we know they involve no variable-substitutions because, by premise, $t_{1}$ did not.

Lemma 4.3. For any localizing-condition $C_{l o c}$ and resolution-tree $t_{1}$ that involves no variable-substitutions, we can construct a localized-form of $t_{1}$.

Proof. If $t_{1}$ has one node which does not satisfy $C_{l o c}$, then it must have some sub-tree (proper or not) $s t_{1}$ whose only $C_{l o c}$-violating node is its root. From Lemma 4.2, we know that we can construct a localized-form $s t_{2}$ of $s t_{1}$ that has no variable-substitutions. Given Lemma 4.1, if we replace $s t_{2}$ with $s t_{1}$, we will end up with a tree $t_{2}$ whose root is associated to the same clause as that of $t_{1}$, whose leaf-nodes are associated to a set of clauses that is a subset of that of $t_{1}$, and that has strictly fewer nodes that do not satisfy $C_{l o c}$. Clearly, $t_{2}$ will also involve no variable-substitutions. We can continue iteratively doing this, which will eventually arrive us at a localized-form of $t_{1}$.

\section{A localization theorem}

Theorem 1. (localization) We can construct a localized-form for every resolutiontree and localizing-condition.

Proof. Consider an arbitrary resolution-tree $t_{1}$ and localizing-condition $C_{l o c}$ - Let $t_{2}$ be the complete-concretization of $t_{1}$. Given Lemma 4.3, we can construct a localized-form $t_{3}$ of $t_{2}$. We can then form an abstract-rebasis $t_{4}$ of $t_{3}$ using the set of clauses associated to the leaf-nodes of $t_{1}$. Clearly, $t_{4}$ 's root node and leaf-nodes will bear the needed relation to those of $t_{1}$. What remains to be shown is that $t_{4}$ 's nodes all satisfy $C_{l o c}$. Given that $t_{4}$ is abstractly-equivalent to $t_{3}$, and all of $t_{3}$ 's nodes satisfy $C_{l o c}$, Lemma 3.1 implies that all of $t_{4}$ 's nodes will satisfy $C_{l o c}$ as well.

\section{New proof of Craig's Interpolation Theorem}

There are variations in the literature on how to formulate the theorem originally stated and proven by William Craig [2], but the below version will best suit our purposes.

Theorem 2. (Craig Interpolation) If a statement $A$ entails a statement $C$, then there is some statement $B$ such that $A$ entails $B, B$ entails $C$, and every predicate and constant term appearing in $B$ appears in both $A$ and $C$.

Proof. If $A$ entails $C$, this implies that $(A \& \neg C$ ) is not satisfiable. Given that resolution derivation is refutation-complete [1], this means that there exists a resolution derivation whose basis (set of axiomatic clauses) $C L_{1}$ is a conjunctivenormal-form representation of $(A \& \neg C)$, and which ends in the empty clause. Let $t_{1}$ be a resolution-tree representing this derivation.

For each constant that appears in $A$ but not $C$, replace it in the tree with a unique skolem-constant (as though the constant was an existentially quantified 
variable). It can be seen that this will produce a valid resolution-tree $t_{2}$ ending in the empty clause where :

- The axiomatic basis of $t_{2}$ is a clausal representation of $\left(A_{1} \& \neg C\right)$, where

- $A_{1}$ is an entailment of $A$

- all of $A_{1}$ 's terms appear in $A$

- all constants in $t_{2}$ appear in $C$

Let $S P_{1}$ be the set of predicate-terms that are in $A$ but not in $C$. Let $C_{\text {org }}$ be the condition that is satisfied by any leaf-node including a term in $S P_{1}$, or any non-leaf node which is such that it involves an elimination in $S P_{1}$, and so do all of its non-leaf-node descendants. It can be seen that $C_{\text {org }}$ is an organizingcondition. Let $C_{l o c}$ be the relevant localizing-condition defined in terms of $C_{\text {org }}$ - Given Theorem 1 (localization), we can construct a localized-form $t_{3}$ of $t_{2}$ for $C_{l o c}$. Consider the set $N_{1}$ of nodes in $t_{3}$ that involve an elimination in $S P_{1}$ but whose parents do not. It can be seen that :

- none of the clauses associated to nodes in $N_{1}$ include predicate-terms in $S P_{1}$

- the set of leaf-nodes with terms in $S P_{1}$ is exactly the set of leaf nodes descendant of nodes in $N_{1}$

- the set of leaf nodes descendant of nodes in $N_{1}$ together represent a statement $A_{2}$ where

- $A_{1}$ entails $A_{2}$

- $A_{2}$ 's terms are all in $A$

If necessary, modify the clauses in the non-leaf inclusive-descendants of $N_{1}$ so that each step is the most general unifier of its children. It can be seen that this preserves the validity and result of the tree, and that now :

- The constant terms in the set of inclusive-descendants of members of $N_{1}$ all appear in $A$.

- And so the set of clauses assigned to $N_{1}$ corresponds to a statement $A_{3}$ such that :

- $A_{2}$ implies $A_{3}$

- $A_{3}$ 's terms are all in both $A$ and $C$

Let $N_{2}$ be the set of leaf-nodes that are not descendant of members of $N_{1}$ , and yet which correspond to part of the clausal representation of $A$. Clearly their associated terms are all in $A$. Since the tree as a whole only includes constant terms from $C$, they will not include any constant terms not in $C$. And since all the leaf nodes with terms in $S P_{1}$ are descendant of $N_{1}$, they will not 
include any predicate terms not in $C$. And so the set of clauses assigned to $N_{2}$ will only include terms which appear in both $A$ and $C$.

If we then consider the set of nodes $\left\{N_{1} \cup N_{2}\right\}$, we see that the set of clauses assigned to them corresponds to the representation of a statement $A_{4}$ which is an implication of $A$, and whose terms all appear in both $A$ and $C$. Remove all the nodes that are descendant of $N_{1}$, and we are left with a resolution-tree operating over a basis that is a clausal representation of $\left(A_{4} \& \neg C\right)$, and which ends in the empty-clause. This shows that $A_{4}$ entails $C$. And so $A_{4}$ is our desired $B$.

\section{References}

[1] Alan J. Robinson. "A Machine-Oriented Logic Based on the Resolution Principle". In: Journal of the ACM 12.1 (1965), pp. 23-41. DOI: 10.1145/ 321250.321253.

[2] William Craig. "Three uses of the Herbrand-Gentzen theorem in relating model theory and proof theory". In: The Journal of Symbolic Logic 22.3 (1957), pp. 269-285. DOI: $10.2307 / 2963594$. 